\title{
Probiotics in gastric and intestinal disorders as functional food and medicine
}

\author{
Nils-Georg Asp, Roland Möllby, Lisa Norin and \\ Torkel Wadström
}

The chairman of the first session, Tore Midtvedt, opened the conference by reminding the audience that since ancient time, food, bowel and health have been interrelated medical topics. Microorganisms, especially lactobacilli and some yeasts, have been used as health-promoting agents since ancient time. Metchnikoff suggested that the long healthy life of Bulgarian peasants resulted from consumption of fermented milk products. The term "probiotic" is still under debate. In all definitions there are two prerequisites: 1) the microbes have to be alive, and 2) the microbes are given for other than nutritional reasons, i.e., it is not the caloric or nutrient content of the probiotics that counts. ME Sanders' definition from 1996 says that probiotics, simply defined, are microbes consumed for health effects. The term probiotics is used in food applications, and biotherapeutics is often used in clinical applications. In selecting probiotic/biotherapeutic strains, Midtvedt emphasised the importance of thinking function rather than disease, and of investigating functions in specified compartments of the body. For instance, $\beta$ glucuronidase activity of the colonic microflora may modify conjugation of endo- and exogenous substances, and the presence of cytochrome P 450 in human intestinal flora may influence the expression of hepatic cytochrome P 450.

\section{The gastrointestinal microflora}

James Versalovic presented an overview of the intestinal microflora. A number of Lactobacillus species colonise the stomach and intestine immediately after birth, adhere to epithelial cells and become a part of the stable intestinal flora during development and adulthood. The genus Lactobacillus includes more than 60 different species, and has received attention as a source for probiotic agents and protein delivery systems for the mammalian intestine. Only few species have been de- scribed as the dominant indigenous lactobacilli of the human intestine. From 130 intestinal biopsies the following lactobacilli were cultured: $38 \% L$ gasseri, 20\%, L fermentum, $8 \%$ L crispatus, 7\% L rhamnosus, $4.4 \%$ L salivarius and $4.4 \%$ L mucosaelike probiotic strains (unpublished data). Versalovic stressed that there is a demand for a more detailed understanding of the composition of the commensal intestinal microbiota and their interactions with the intestinal immune system, which would create breakthroughs in probiotic research. Gene and protein discovery efforts with defined clones of specific intestinal lactobacilli and other lactic acid bacteria would lead to the unravelling of mechanisms of probiosis and development of new therapeutic strategies with such probiotic bacteria. These could be achieved by

- A detailed mapping of lactic acid bacteria in the mammalian intestine

- Clone-based investigations of commensal microbes and of the functions of different probiotics

The question is raised whether one can alter or restore the microbial balance in human individuals. And does restoration of a microbial balance lead to restoration of an immunologic balance?

Göran Molin reviewed recent work on the intestinal microflora, in collaboration with B. Jeppsson, $S$. Ahrné and M. Wang. By the use of phenotypical identification methods and anaerobic culture techniques, the GI-flora has been reported to be dominated by genera such as Bacteroides, Eubacterium, Clostridium, Ruminococcus, Peptococcus, Peptostreptococcus, Bifidobacterium and Fusobacterium. However, culture-based methods provide an incomplete assessment of the bacterial GI-flora and phenotypic features are not always reliable or 
sufficiently descriptive in bacterial systematics. Sixty to eighty percent of the GI-bacteria have been claimed to be unculturable. One way to circumvent the need for cultivation is the use of DNA amplification by PCR and subsequent cloning of 16S rRNA genes, followed by comparisons with available databases. Microbes that are in close contact with the body are more likely to interact with human physiology, and this part of the flora of the GI tract may be involved in developing chronic inflammation and colon cancer. It can also be affected by antibiotics, cytotoxic drugs and radiotherapy, trauma, and intensive care therapy. It is thus important to map the mucosa-associated flora in healthy individuals in order to clarify the pathogenic risk under stressed and other disturbed conditions in man, before one tries to perform similar studies in diseased subjects.

\section{Dyspepsia, gastric reflux disease and gastric cancer}

F. Bazzoli introduced this subject by stating that epidemiological investigations in the US show that $25 \%$ of the population suffer from recurrent dyspepsia, but less than 50\% seek medical treatment for their symptoms. These symptoms have, however, a significant impact on the quality of life. Dyspepsia is defined as pain or discomfort from the upper abdomen. In practice, there is considerable overlap of dyspepsia and gastro-oesophageal reflux disease (GORD/GERD). A key element to effectively managing patients with dyspeptic symptoms is the identification and exclusion of GORD through proton pump inhibitor (PPI) therapy to dyspeptic persons. These are individuals who are exposed to the risk of physical complications or who experience clinically significant impairment of health-related well-being due to episodes of GORD (after reassurance). A probiotic treatment approach in relation to GI disorders, aiming to restore or alter/balance the intestinal microflora, might have an anti-inflammatory effect, could reduce gas production, could promote intestinal motility and could have a visceral analgesic effect. Moreover, by including prebiotics, a proximal colon dysfunction might be corrected via carbohydrate fermentation. The proximal gastric tone could also be inhibited with a role in the regulation of gastric motility.

Lars Lundell described GORD as "a burning feeling rising from your stomach or lower chest up towards the neck". There is one major concern with dyspepsia - an increased risk with time of develop- ing oesophageal adenocarcinoma. How to reduce the discomfort/problems in dyspepsia subjects? The most important way to achieve improvement is by a lifestyle modification, e.g. by reducing use of tobacco, alcohol and coffee, and weight reduction by an overall dietary alteration. Patients with a Helicobacter pylori (H.p) infection were formerly also included in this group. Thus $25-30 \%$ of middleaged patients with earlier diagnosed reflux problems have been identified as H.p patients. After eradication of the infection, there was surprisingly a significantly higher risk of GORD symptoms in one study, but the disease signs had almost totally disappeared two years after the H.p eradication in a group of almost 1700 subjects, and the risk of relapse was also strongly reduced. Lundell's overall conclusion was that the benefit of eradicating H.p in non-ulcer dyspepsia and GORD is greater than the possible risks.

The state of the art regarding probiotics and Helicobacter pylori gastritis was reviewed by $A$. Gasbarrini, whose full paper is published in this issue of the Scandinavian Journal of Nutrition (pp. 26-31). Proposed mechanisms of action of oral bacteriotherapy include synthesis of antimicrobial substances such as organic fatty acids, ammonia, hydrogen peroxide and bacteriocines, a competitive interaction with pathogens for "space and food", through the use of available nutrients and occupation of microbial adhesion sites, modification of toxins or toxin receptors, and immuno-modulation.

H. pylori is one of the most prevalent infectious agents worldwide and has been indicated as one pathogenic factor in the development of chronic gastritis, peptic ulcer disease and gastric malignancies. One suggested mechanism related to probiotic therapy is that Lactobacilli spp. are supposed to adhere and even transiently reside in the stomach, enhance the immune response and reduce the $H$. pylori inflammation effect on the host gastric mucosa. Furthermore, probiotics have been shown to improve patients' compliance with antibiotic treatment by reducing side effects.

Y. Koga, together with A. Tamura, K. Kobayashi and A. Takagi, presented studies on $H$. pylori inhibition in experimental animals and man. Lactobacilli are the dominant bacteria found in the fasting stomach although their number is small. However, it increases by a factor of thousands in individuals with low gastric acidity, suggesting that the stomach is potentially an organ which lactoba- 
cilli may colonise in high numbers. More than 200 strains of lactobacilli were investigated in order to select strains exhibiting high lactic acid production, efficient detachment of the H. pylori, and strong resistance to gastric acidity.

Lactobacillus gasseri strain OLL 2716 (LG21) was selected for further studies in 5-week-old gerbils. H. pylori were inoculated with LG21 and the animals were followed. After one year, $83 \%$ of the gerbils inoculated with LG21 were without detectable amounts of $H$. pylori, whereas $26 \%$ carried these microbes when the gerbils initially not were inoculated with LG21. Thus, oral administration of LG21 as a probiotic to H. pyloriinfected Mongolian gerbils significantly prevented the incidence of gastritis and gastric ulcers.

Thirty-one human subjects with documented $H$. pylori infection (exclusion criteria antibiotic therapy within less than 1 month) were given $90 \mathrm{~g}$ of yoghurt twice daily for 8 weeks. One week following the completion of the first part (week 9), the subjects were given $90 \mathrm{~g}$ of yoghurt containing more than $6 \times 10^{6} \mathrm{CFU} / \mathrm{g}$ of LG21, twice daily for 8 weeks. Urease tests showed significantly lower values at the end of the study (week 18) than at week 0 and 9 $(\mathrm{p}<0.05)$. However, 3 months later, a reversal was detected, which means that the $H$. pylori were not completely eradicated. A significant decrease in the urease levels was found after LG21 treatment, which indicates a reduction of the H. pylori in the stomach. Moreover, a significant improvement in the degree of gastric mucosal inflammation was demonstrated by analysis of the serum PGI/PGII levels.

Koga's overall conclusion was that orally administered LG21 might efficiently suppress $H$. pylori colonising the human stomach and thus alleviate the inflammation.

Stomach cancer, reviewed by T. Rokkas, is the second most common cancer in the world. In 1996, it was estimated that over 1 million new cases were diagnosed worldwide, accounting for nearly $10 \%$ of all new cancers. Nearly two-thirds of all cases occur in developing countries. Gastric cancer mortality and incidence show a geographical variation with the highest rates found in Japan, Central and South America and eastern Asia. In developed countries rates have steadily declined, with Australia, Canada and the USA showing the lowest rates. Today, stomach cancer rates are 50\% lower in women than in men. Migrants from high- to low-risk countries tend to maintain the high-risk characteristics, whereas the offspring acquire a risk closer to that of the host countries. Low socioeconomic status is also shown to be associated with high risk.

The evidence that diets high in vegetables and fruits protect against stomach cancer is convincing. Summarised evidence suggests that raw vegetables and fruit containing vitamins $\mathrm{C}$ and $\mathrm{E}$, betacarotene and minerals such as selenium protect against stomach cancer. The protective effect of ascorbic acid against gastric carcinogenesis is due to its ability to inactivate reactive oxygen species (ROS, "free radicals") as well as its nitrite scavenging effects.

The suggested association between gastric cancer and $H$. pylori infection is mainly based on the fact that $H$. pylori is the major causative agent of chronic gastritis, developing into atrophic gastritis and intestinal metaplasia, the precursor lesion of gastric cancer. In this process, dietary factors play an important role. However, no direct evidence exists in the literature on the relationship between probiotics and gastric cancer.

\section{Bacterial translocation - impact of probiotics on barrier function}

Bacterial translocation is defined as the passage of bacteria or bacterial products across an intact gastrointestinal tract barrier. Bengt Jeppson reported that there is ample evidence that this occurs in humans, but its role in the pathogenesis of different diseases is unclear. In order for bacterial translocation to be involved in disease processes, there must be elements of both permeability disturbance and reticulo-endothelial dysfunction. Disruption of the intestinal bacterial microflora balance may increase the incidence of bacterial translocation by modifying a normal intestinal barrier function. The area is reviewed in a separate paper in this issue (pp. 37-41). In a number of studies showing positive effects of probiotics there has been an increased risk of secondary bacterial infections of enteric origin, due to the surgical trauma or ongoing infection. By altering the luminal content of bacteria it seems possible to reduce the incidence of secondary infections. Although these results need to be confirmed in larger prospective studies they indicate a new concept of altering the luminal bacterial milieu resulting in reduced secondary infections. The influence of the luminal milieu on bacterial translocation is still 
not fully understood. We have convincing evidence from experimental studies, but so far only circumstantial evidence can be found from clinical studies.

Probiotics in irritable bowel syndrome (IBS)

IBS is a multi-factorial condition that accounts for $20-50 \%$ of referrals to gastro-intestinal (GI) clinics in the UK, said Jennifer Madden. Typical symptoms are excessive flatus, abdominal pain and variable bowel habit, for which no pathological cause can be determined. Suggested aetiologies include psychosocial factors, altered GI motility, heightened sensory function of the intestine and/or malfermentation of foods. Changes in species composition and metabolic activities of the intestinal microbiota may also play a role. Several studies have shown that the onset of IBS is significantly higher after specific event(s) such as an antibiotic treatment, abdominal or pelvic surgery or gastroenteritis. The intestinal flora in IBS has been extensively studied and higher numbers of facultative anaerobes with lower numbers of bifidobacteria and lactobacilli have been found. So far, however, it has not been possible to determine whether an altered intestinal microflora composition is a contributory factor to the aetiology of IBS. Some studies are described in a full paper in this issue (pp. 32-36), using symptomatic differences as a definable endpoint, have shown significant improvements following probiotics, though others are subjective and likely to contain a certain amount of placebo response. Most studies using IBS patients have been on small sample populations and further studies are needed, with either larger patient numbers, or in defined subsets e.g. diarrhoea predominant. Some results suggest a trend towards a preventive, rather than a therapeutic role, for probiotics in the management of IBS.

\section{Probiotics in Inflammatory Bowel Disease (IBD)}

The possible role of probiotics in inflammatory bowel disease (IBD) was reviewed by P.B. Heczko and co-workers. "This area unfortunately contains more hopes and discussions than well performed studies," said Heczko. During the last 3 years there have been 13 reported clinical trials with varying outcomes, but they have given rise to 24 critical reviews. However, data in animals with colon inflammation corroborate that bacterial products, including those of Lactobacillus species, may be involved in the process in two ways: either perpetuating or modifying the course of the inflammation by interactions with mucosal immune cells. A probiotic preparation (VSL\#3) that contains a combination of three species of bifidobacteria, four strains of lactobacilli and one streptococcus strain has shown promise in maintaining remissions in ulcerative colitis and pouchitis, as well as in preventing the postoperative recurrences of Crohn's disease. On the other hand, a well studied probiotic strain Lactobacillus rhamnosus GG, was reported as ineffective in preventing recurrences of CD in two studies done in adults but giving some improvements in children with CD. Although well planned and controlled clinical studies are obviously required to delineate fully the potential for probiotics in IBD, basic studies are even more important to elucidate mechanisms of anti-inflammatory activity of so many different probiotic bacteria.

\section{Probiotic bacteria, infections and allergies}

This section was introduced by a review presentation by M. Mikelsaar on probiotics basis in prevention and treatment of infections and allergy. Microbial interference therapy (MIT) has been applied in many infectious diseases, including travellers' diarrhoea, antibiotic-associated colitis, Clostridium difficile related infections, rotavirus enteritis, urinary tract infections, vaginosis and many other diseases of infectious origin.

Mikelsaar mentioned several mechanisms of action of probiotics: (a) probiotic specific competitive exclusion of infectious agents by anti-adhesion factors, competition for nutrients, antimicrobial metabolites, bacteriocins, suppression of toxin production, altogether sustaining the balance of the microflora; (b) probiotic and host-specific immune response towards foreign antigens of microbial origin by immune modulation, enhancing the activity of macrophages, increasing the amount of natural killer (NK) T-cells and the level of interferon gamma, stimulating the production of antibodies; (c) induction of some protective factors such as cytokines, mucus, SCFA and defensins against pathogens in epithelial cells.

Allergy is defined as an inflammatory disease resulting from abnormal reaction to some innocuous antigen. The prevalence of allergic diseases such as asthma, rhino-conjunctivitis and atopic dermatitis has increased over the past decades worldwide. Several clinical trials with probiotic administration have been conducted. The majority can be divided into two groups: allergy preventive 
and treatment trials. Recent investigators have distinguished the sensitisation phase of allergy, the phase of allergic manifestations and its downregulation phase. Several cross-sectional and prospective studies have shown pronounced differences in the establishment of both lactobacilli and other intestinal microbes in allergic as compared to nonallergic children, and Mikelsaar concluded that studies to create an adequate scientific basis of probiotics would be needed to start trials with hostoriented and well-targeted functional foods for prevention and treatment of different inflammatory disorders.

Studies investigating probiotics in infectious diarrhoea in children were reviewed by Merja Ashorn. Rotavirus gastroenteritis in Finland account for 3\% of all children hospitalised between 1985 and 1995, and in general, rotavirus diarrhoea accounts for over $50 \%$ of all cases of gastroenteritis in Finland. The mean hospital stay due to paediatric diarrhoea was 3.3 days in 1985 vs. 2.3 days in 1995 . One way to minimise this disease has been to use probiotics.

Adherence of some probiotic bacterial strains to human intestinal mucus in infants in relation to rotavirus infection has been tested in vitro, using Lactobacillus GG, L. casei Shirota, L. paracasei F19, L. acidophilus and Bifidobacterium lactic Bb12. Adhesion to mucus, isolated from patients one month after virus infection, was strongest with $B b 12$ and LGG, and strains proven to shorten the duration of rotavirus diarrhoea are $L G G, L$. reuteri, L. casei Shirota and Bifidobacterium lactis Bb 12.

A review published by Szajewska and Mrukowicz (1) concluded that $L G G$ consistently reduced the duration of diarrhoea in children as compared to placebo. Van Niel et al. (2) showed that in 9 randomised studies, $L G G, L$. reuteri and $L$. acidophilus/L. bulgaricus reduced diarrhoea duration of 0.7 days and the frequency of 1.6 stools/day. A dose response relationship was observed: optimal dose $>10^{10} \mathrm{CFU}$ during the first 48 hours. Guandalini et al. (3) investigated an acute onset of diarrhoea in children 1 month to 3 years of age, and he showed that in group $\mathrm{A}$, in which the children got oral rehydration and placebo, the mean duration of diarrhoea was 72 hours as compared to 56 hours in group B $(\mathrm{p}<0.008)$ which got rehydration + LGG therapy. In another placebo controlled study of Peruvian undernourished children (4), there was a significantly decreased amount of adenovirus gastroenteritis in the LGG group.
In the future, there is a need to expand studies on therapeutic and prophylactic use of probiotics in diarrhoea, especially in bacterial diarrhoea and in developing countries. This can be done by e.g., searching for strains with optimal therapeutic potential in humans, including the optimal dose, by developing better ways to measure colonisation in humans, and by investigating mechanisms of action, including immune stimulation. The final aim is to obtain probiotics that promote normalising of the flora and thereby enhance the healing of small bowel mucosa.

The last presentation in the section on probiotic bacteria, infections and allergies was given by $A$. Wold. The small intestine contains relatively few bacteria. An intake of $10^{10}-10^{11}$ bacteria per day via the food is probably sufficient to significantly influence the gut-associated immune system. Bacteria are taken up by $\mathrm{M}$ cells covering the Peyer's patches and are processed by macrophages and dendritic cells in these patches, leading to stimulation of innate immunity and specific immune responses to the bacteria. Monocytes react differently to Gram-positive and Gram-negative bacteria, i.e. Gram + organisms elicit more of IL12 and $\mathrm{TNF} \alpha$, while Gram- organisms give rise to relatively more IL6, IL8, IL10 and PGE2. The former response stimulates a cell-mediated immune reaction and a concomitant macrophage activation, while the latter induces a maturation of dendritic cells and antibody production.

As reviewed above, probiotics have been convincingly shown to shorten acute watery diarrhoeal episodes in children, mainly those caused by rotavirus. Such diarrhoea episodes are self-limiting and of short duration. Wold thought that it is not very likely that this has much to do with specific immune responses to the rotavirus, since these appear later. Rather, one may assume that the effect of bacteria and their products on cells of the innate immune system leads to changes in secretion, permeability and motility via effects on cytokines, hormones and the function of the enteric nervous system.

Probiotics have also been shown to shorten $C$. difficile-induced diarrhoea. Such diarrhoea is elicited in the large intestine through the action of $C$. difficile toxins. The mechanism by which probiotics counteract $C$. difficile-induced diarrhoea is not known. The most convincing effect was shown using the yeast Saccharomyces boulardii, an organ- 
ism that does not colonise the large intestine. In some way, probiotic intake must change the milieu in the large intestine so that the clostridia do not thrive, and produce their toxins, or that the intestinal mucosa becomes less sensitive to the toxin.

Probiotics have been tried to treat or prevent allergy. A large study by Trapp and co-workers (5) suggested that live or inactivated yoghurt might reduce asthma symptoms. Whereas yoghurt versus inactivated yoghurt treated groups were randomised, subjects not eating yoghurt were those who refused to take yoghurt, which limits the conclusions from the study. Wheeler and co-workers (6) found no effect in 15 patients with moderate asthma of yoghurt with lactobacilli. Lactobacillus GG has been tried both as treatment of cow's milk allergy in young children and as a means to prevent occurrence of allergy $(7,8)$. A reduction of eczema, but not IgE antibodies, has been detected in these studies. No effect of probiotics was seen on hay fever or on respiratory allergy. The mechanism behind the anti-eczematous effect is unknown, but there is nothing to indicate that probiotics reduce IgE production through any immuno-regulatory mechanism. The effect might instead be on gut permeability and inflammation and possibly, in addition, on the local conditions in the skin.

Wold ended by asking if the increased frequencies of diseases like IgE-mediated allergies, Type 1 diabetes, inflammatory bowel diseases and multiple sclerosis could be related to "microbial deprivation disorders". There are several possibilities for a dysfunctional microbial stimulation caused by hygienic measures in western societies ("The hygiene hypothesis"):

- Some key groups of microbes may have disappeared

- Some other group of microbes with untoward effects may have increased due to lack of competition

- The turnover of strains in the microflora may be too low to generate a sufficient number of activation events for the immune system

\section{Probiotics and food-based vaccines}

This subject was presented by $P$. Michetti. The immuno-modulatory capacity of probiotics has stimulated the use of lactobacilli, streptococci, lactococci, Bacillus spores, and Saccharomyces as vaccine adjuvants, as well as vehicles for antigens for active immunisation. Probiotics have been experimentally used for parenteral immunisation, but their most promising use is for oral delivery of immunoglobulin-like molecules or vaccination.

A great variety of effects of probiotic strains on the immune system may be used to elicit different immune response, depending on the disease to be prevented, and various probiotics may deliver antigens in specific compartments of the immune system. In addition to express vaccine antigens, these microorganisms are now further modified to co-express interleukins and other immunomodulatory molecules. Evidence is accumulating that probiotic-based vaccine and drug delivery strategies are efficacious in animal models. For example, IL10 producing Lactococcus lactis have been shown to cure experimentally induced colitis in IL-10 deficient mice. Evidence is awaited to determine whether these strategies can be applied in human beings.

In the search for cheap production of recombinant vaccines for oral use and to facilitate specific vaccination procedures, vaccine antigens, combined with adjuvants, have been produced in edible plants, especially in potato tubers. These approaches may be of particular interest for developing countries where more expensive vaccines are not optimally available. Whether living microorganisms are given to humans or recombinant crops are grown for recombinant protein production, uncontrolled release of genetically-modified organisms will occur if these vaccine strategies are applied in medical practice. Such a release has already elicited major concerns about and opposition to food products, which will apply to vaccine-related organisms. In addition, specific vaccine-related problems may have to be considered. Indeed, unplanned vaccination may occur, with possible adverse events, and repeated exposure to vaccine antigens may induce oral tolerance.

\section{The safety of probiotics}

The assessment of the safety of probiotics is an important part of their characterisation for human use. The presentation on this subject by $S$. Salminen is presented as a separate article (pp. 42-48).

It is essential that a probiotic does not have the ability to invade the host intestinal tissue and cause an infection. Probiotic organisms must be sensitive to broad-spectrum and commonly used antibiotics. 
This is a significant issue where the intestinal barrier is immature as in infants, and where its integrity is impaired by radiotherapy, antibiotic treatment or disease; and in immuno-compromised states, such as human immunodeficiency virus (HIV) infection. Epidemiological surveillance studies have reported extremely rare cases of bacteraemia with lactic acid bacteria and bifidobacteria. The cases were not associated with probiotic use and were often preceded by serious underlying diseases and previous antibiotic-treated bacteraemia. Studies on probiotic use by immuno-compromised subjects together with the epidemiology studies support the safety of current probiotics.

Correct taxonomic identification of both species and strain is a safety issue for quality control of the product, consumer and prescriber information, diagnosis and appropriate treatment of suspected clinical cases, as well as for epidemiological surveillance of the exposed population. Spore-forming bacteria is a special issue, where rigorously designed studies are needed to characterise and demonstrate both the efficacy and safety of the probiotic microorganism.

The history of safe use of food microorganisms has been reviewed recently specifically for dairy foods through the International Dairy Federation. As a result a list of cultures with safe history of use has been produced. Especially rigorous procedures are required for novel organisms and for GMO microbes.

\section{Claims and their substantiation}

Products with probiotics as active ingredient can be either "functional food" or medicine, depending on product characteristics - food or tablet, capsule, powder - as well as intended use and claims in labelling and marketing. G. Reid, presenting the International Scientific Association for Probiotics and Prebiotics (ISAPP), mentioned the fact that there are websites around with ridiculous claims by companies with unreliable products containing strains of dubious and unproven properties.

ISAPP exists to foster high-quality scientific information for the probiotic and prebiotic fields and to provide guidance for collaborative and multidisciplinary research. ISAPP's intent and hope is that this information will be translated by industry into high-quality, healthful products which benefit people all over the world (www.isapp.net).
Regardless of whether probiotic products are food products or medicines, effect documentation in human clinical trials is essential. A. Melander stressed that every medical intervention (lifestyle modification, surgery, radiation, pharmacotherapy or probiotic therapy) should be based on genuine and confirmed evidence of patient benefit. Moreover, the benefit should be greater than the risks. When evaluating the benefit, and the benefit/risk ratio, it is important to consider the aim of the treatment: Is it supposed to be curative, to substitute, to alleviate symptoms, or to prevent future disease or complications? The number of subjects and the duration of the clinical trial needed to evaluate benefits and risks differ markedly between these situations. This was illustrated by examples from the pharmaco-therapeutic area. It is also important to consider the difference between evidence of efficacy (i.e. the potential of a drug as shown in a randomised, controlled clinical trial), effectiveness (i.e. in the epidemiological/observational, real-life situation of routine treatment) and efficiency (i.e. when cost effectiveness is also included).

Curative drugs, with penicillin as the prime example, are rather few. Due to their curative nature, such drugs are given for a short period of time. Hence, their efficacy is usually easy to evaluate in short-lasting controlled trials, in which the control is either placebo or an already established standard drug. Also the effectiveness may be ascertained in short-term observational analyses. However, the safety aspect may not be sufficiently elucidated by short-term studies, as witnessed by the drastic increase in bacterial resistance against most antibiotics. Recent studies have shown that the extent of resistant pathogenic bacteria correlates with the extent of exposure to various antibiotics not only in the individual but also at the population level. This development was not foreseen for several decades after the introduction of antibiotics in general practice.

Proton pump inhibitors (PPI), such as omeprazole, were mentioned by Melander in this context, as they not only alleviate symptoms of gastric hyperacidity and gastro-duodenal ulcers but even help to enhance the rate of ulcer healing. On the other hand, there is reason to assume that they may also enhance ulcer relapse rate and may favour reinfection with Helicobacter pylori. Moreover, a rebound effect (hypergastrinaemia) may lead to a 
situation of PPI habituation and dependence. As in the case of antibiotics, this emphasises the importance of long-term evaluation of all potential consequences even of short-term interventions. Therefore, evaluation of the anti-Helicobacter efficacy and effectiveness of probiotics seems highly important.

Substituting drugs may, like curative drugs, allow a normal life, although on condition that the substitution is continuous, e.g. as with insulin in type 1 diabetes, thyroid hormone in hypothyroidism and corticosteroids in Addison's disease. The evaluation of substitutive drug efficacy and effectiveness is usually easy; without insulin the patient with type 1 diabetes will rapidly become ill and die. This would apply also to probiotics, if potentially lifesaving agents of this kind become available.

Symptom-alleviating drugs are predominant in pharmacotherapy, including analgesic, anxiolytic, hypnotic, antiepileptic, neuroleptic, antidepressant, anti-Parkinson, anti-allergic, antacid and several other drugs. The patient's self-rating of symptoms (less pain, easier movements, less depressed, etc.) is central in the evaluation of efficacy and effectiveness of such drugs. However, controlled studies involving placebo or an established standard drug are necessary, as placebo/nocebo effects often are pronounced. This should be applicable to present or future symptom-alleviating probiotics as well.

Preventive drugs are rapidly increasing in both number and use, and the pharmaceutical industry invests more resources in this area than in any other. Such drugs, by definition, are to be taken for many years, if not life-long, and because they may be indicated for use by large segments of the population. Prime examples are blood pressure lowering, lipid-lowering, glucose-lowering and weight-reducing drugs. The purchase costs of the two former drug groups already comprise more than $10 \%$ of the current total drug purchase costs in Sweden.

Evaluation of the efficacy of preventive drugs presents a much more laborious, time-consuming and expensive task than that of other drugs. This is because the aim is to reduce morbidity and/or mortality, necessitating studies lasting several years and including large numbers of patients. Moreover, the patients ought to be representative of major segments of the population, including different groups of age, sex, ethnicity, co-morbidity and concurrent treatment. However, this has only rarely been the case even in latter-day studies. Further- more, it is important to analyse not only the relative, but also the absolute efficacy, preferably expressed as numbers of individuals needed to treat (NNT) in order to prevent one death or other major event.

Studies of the effectiveness of preventive drugs, subsequent to those of efficacy, are particularly important for several reasons. For practical and economic reasons, even the best of efficacy studies have been limited to a few years and some thousand subjects. Hence, the representativity of the trial is often insufficient concerning long-term patient benefit. Moreover, long-term or unusual but serious risks, e.g. cancer, may be missed in trials. In addition, patient adherence to treatment is commonly low, especially in long-term, preventive treatment. In addition, interactions with other drugs may occur in routine treatment. Melander concluded that, particularly as long-term trials of probiotics used in disease prevention seem far away, it is important to initiate effectiveness studies, i.e. epidemiological-observational ones, on probiotics employed to prevent disease and disease complications.

\section{The legal framework for "functional food"}

Different types of food products, relevant for probiotics, were reviewed by $\AA$. Bruce. There are a number of legally defined subgroups of foods which are identified and characterised by various national and international regulations:

- Foodstuffs intended for particular nutritional uses

- Novel foods

- Fortified foods (with added vitamins and/or minerals)

- Dietary supplements (tablets etc. with vitamins and/or minerals or /in future/ with other compounds)

- (Functional foods - not legally defined in the EU or by Codex Alimentarius)

Two types medicinal products are also relevant:

- Natural remedies

- Ordinary medicinal products

Foodstuffs for "Particular Nutritional Uses" are intended for certain categories of persons who are in a special physiological condition or whose digestive processes or metabolism are disturbed. 
A new subgroup is "Dietary foods for special medical purposes", products intended to meet the particular nutritional requirements of persons affected by or malnourished because of a specific disease, disorder or medical condition.

Novel Foods follow the Regulation of the European Parliament and of the Council of 27 January 1997. The regulation applies to the placing on the market of foods or food ingredients which have not hitherto been used for human consumption to a significant degree in the EU and which fall under the following categories of foods and food ingredients:

- containing or consisting of genetically modified organisms (GMO)

- produced from, but not containing, GMO

- with a new or intentionally modified primary molecular structure

- consisting of or isolated from microorganisms, fungi or algae

- consisting of or isolated from plants or animals, except for foods and food ingredients obtained by traditional propagation and breeding practices and which have a history of safe use

- to which has been applied a production process not currently used, where that process gives rise to significant changes in the composition or structure of the foods or food ingredients which affect their nutritional value, metabolism or the level of undesirable substances.

Natural remedies in Sweden are medicinal products whose constituents have natural origins, and which are parts of an animal or a vegetable, $a$ bacterial culture, a mineral, salt or salt solution. The product must be suitable for home cures in accordance with well-proven Swedish tradition, or traditions in other countries considered to have medicinal traditions similar to those in Sweden. The Medicinal Products Agency should approve any new product.

International and national activities. The matters of functional foods and health claims about foods have been more and more discussed during the last decade within a number of international contexts. The European Commission stated, in a "White Paper on Food Safety" published at the beginning of 2000, that an investigation is to be carried out on this matter during the first years of this decade, and it started in fact in 2002. This summer a new
"Proposal for a regulation of the European Parliament and of the Council on nutrition and health claims made on foods" [COM (2003) 424 final 2003/ 0165 (COD)] was presented.

Codex Alimentarius has dealt with these issues in the labelling committee as well as in the committee on nutrition and foods for special dietary uses. For the time being their definitions are not entirely in agreement with those of the EU.

\section{Japanese perspectives}

The functional foods concept emerged in Japan in the 1980s. T. Sasaki reviewed the current status of health claims for functional foods in Japan. It is widely recognised that food has both a nutritional function (primary function) and a palatability function (second function). In Asian countries such as China, Korea and Japan, people have long believed that food has an additional function, namely the ability to prevent disease (third function). This idea came from the traditional belief that food and medicine come from the same origin and serve the same purpose.

A terminology for "functional food" first arose when an ad hoc research group in Japan began a relevant study in early 1980s. The third function of food was scientifically evaluated by the group with a large grant from the Ministry of Education, Science and Culture, Japan. The International Life Science Institute of Japan (ILSI Japan) has also contributed greatly to the establishment of this new concept by publishing reports and organising domestic and international meetings. ILSI Japan together with 26 food companies will set up a laboratory at the University of Tokyo this year and begin research on nutrigenomics in order to clarify physiological functions of foods.

In 1991, the Ministry of Health and welfare, Japan, issued the "Labelling Regulation for Foods for Specified Health Use (FOSHU)" and Japan was thus the first country that formally accepted the "functional food" concept. The FOSHU-approved foods containing oligosaccharide created a new market in Japan. This market increased greatly when two yoghurt products were approved as FOSHU in 1997. The number of approved FOSHU products is 369 (June 2003) and the market is growing (about 2,000 million euro in 2002). The FOSHU system is fairly well-known to Japanese and consumers easily recognise FOSHU products by the certification mark along with the general 
description of the health claims. It should be noted, though, that in China 3,357 products are approved (March 2002).

Among the 369 products approved so far, 57 are yoghurts or fermented milks, which amount to more than $60 \%$ of the sales in 2002 . This clearly shows how probiotics are important in the FOSHU market. The approved probiotics include six Lactobacillus spp., one Streptococcus sp. and three Bifidobacterium spp. The claims allowed on these 57 products with probiotics are restricted to "promoting the maintenance of good intestinal environment". Other allowed claims for probiotic yoghurts are: "helps increase intestinal bifidobacteria and lactobacilli. This promotes the maintenance of a good intestinal environment and regulates the GI condition", "helps regulate the balance of intestinal microflora which leads to the maintenance of a good GI condition", "helps improve intestinal environment and regulates the GI condition". However, current and future research will clarify the specific health-promoting effects of probiotics beyond basic maintenance of a healthy intestinal environment. Approval of additional health claims in the near future is desirable as scientific evidence amounts.

Sasaki concluded that consumers expect more applications in health-promoting foods, especially as preventive agents of "life-style related diseases". In order to improve public health, it would be desirable to extend the concept of "probiotics" to those bacteria which are effective in promoting healthy conditions in the oral cavity, throat, stomach or vagina. These should be regarded as probiotics, if the scientific evidence is clear. Furthermore, there is a need for international agreements on definitions and permitted claims in this area.

\section{Discussions and conclusions}

The final discussion, opened by Director General Gunnar Alván, Medical Products Agency, Uppsala, Sweden, was focused on documentation of effects of probiotics as functional foods and medicinal products. All agreed that effects claimed in the marketing have to be based on a solid scientific basis, but is it reasonable - and possible - to require the same placebo control and GCP (Good Clinical Practice) standard for functional foods as for medicine? A number of differences between foods and medicines are relevant in this context:
- Food studies cannot always be blinded (although in the case of probiotics true placebo products without the active probiotic microorganism can usually be made enabling double blind studies)

- Foods provide energy and nutrients and hedonic value for money, in addition to the specific health effect, whereas the one and only value of a medicine is its health-promoting or symptomreducing effect

- When provided in a food, the active component cannot be excessively overdosed due to the volume, caloric content and satiety effects of a food

- Costs for good clinical studies with foods are often regarded as too high and difficult to pay back

The need for markers useful as surrogate endpoints in effect studies was stressed. The EU-concerted action project PASSCLAIM (Procedure for the Assessment of Scientific Support of Claims) has a focus on markers, as reported by Nils-Georg Asp (http://europe.ilsi.org/passclaim). With the exception of some established markers such as serum cholesterol/LDL cholesterol level, effect studies with probiotics generally have to measure real end-points such as symptom reduction in irritable bowel-like conditions, allergic manifestations or signs of infection such as diarrhoea or common cold. The validation of markers such as parameters reflecting the immune system and inflammation is an important issue for further research.

A bottom line in documentation is the correct labelling of genus, species and count of viable probiotic microorganisms. A recent survey on the European market has shown that many products do not meet such basic criteria.

The possibility to define general criteria for a "probiotic microorganism/product" - such as survival of gastrointestinal passage, adherence, colonisation, effects on colonic $\mathrm{pH}$ etc. - was discussed. The general opinion, however, was that such criteria would not be meaningful, since the physiological effects of probiotic microorganism are species- and strain-specific, and may even be clone-specific. However, Bengt Björkstén stressed the importance of making studies also with wild strains - for instance in relation to allergy in children, which is much less common in the Baltic countries than in Scandinavia, and related to the occurrence of 
Lactobacilli in the colon. Ideally, studies of specific probiotic microorganisms should have placebos both without the microorganism and with a comparable wild strain. The issue is further complicated by the fact that also dead microorganisms seem to have some physiological effects, e.g. on the immune system.

A general conclusion was that there is intense research with many indication of health effects of probiotics, promising for the further development of both functional foods and medicinal products. However, there is a great need for further well-controlled studies in both patients with various diseases and healthy individuals to explore further the health-promoting potential of probiotics.

\section{Selected references}

1. Szajewska H, Mrukowicz. Probiotics in the treatment and prevention of acute infectious diarrhea in infants and children: a systematic review of published randomized, double-blind, placebo-controlled trials. J Pediatr Gastoenterol Nutr 2001; 33(Suppl. 2): S17-25.

2. Van Niel CW, Feudtner C, Garrison MM, Christakis DA. Lactobacillus therapy for acute infectious diarrhea in children: a meta-analysis. Pediatrics 2002; 109: 67884.

3. Guandalini S, Pensabene L, Zikri MA, Dias JA, Casali LG, Hoekstra $\mathrm{H}$ et al. Lactobacillus GG administered in oral rehydration solution to children with acute diarrhea: a multicenter European trial. J Pediatr Gastoenterol Nutr 2000; 30: 54-60.

4. Oberhelm RA, Gilman RH, Sheen P, Taylor DN, Black RE, Cabrera $\mathrm{L}$ et al. A placebo-controlled trial of Lactobacillus GG to prevent diarrhea in undernourished Peruvian children. J Pediatr 1999; 134: 15-20.

5. Trapp CL, Chang CC, Halpern GM, Keen CL, Gershwin ME. The influence of chronic yoghurt consumption on populations of young and elderly adults. Int J Immunother 1993; IX: 53-64.

6. Wheeler JG, Shema SJ, Bogle ML, Shirrell MA, Burks AW, Pittler A et al. Immune and clinical impact of Lactobacillus acidophilus on asthma. Ann Allergy Asthma Immunol 1997; 79: 229-33.

7. Majamaa H, Isolauri E. Probiotics: A novel approach in the management of food allergy. J Allergy Clin Immunol 1997; 99: 179-85.

8. Kalliomäki M, Salminen S, Arvilommi H, Kero P, Koskinen $\mathrm{P}$, Isolauri E. Probiotics in primary prevention of atopic disease: a randomised placebo-controlled trial. Lancet 2001; 357: 1076-9.

\section{Chairs and speakers}

Gunnar Alvan, Uppsala, Sweden (gunnar.alvan@mpa.se), Merja Ashorn, Tampere, Finland(merja.ashorn@uta.fi), NilsGeorg Asp, Lund, Sweden (asp@snf.ideon.se), Franco Bazzoli, Bologne, Italy (bazzoli@alma.unibo.it), Bengt Björkstén, Stockholm, Sweden (bengt.bjorksten@cfa.ki.se), Åke Bruce, Uppsala, Sweden (akbr@slv.se), Åke Danielsson, Umeå, Sweden (ake.danielsson@medicin.umu.se), Antonio Gasbarrini, Rome, Italy (angiologia@rm.unicatt.it), Lennart Hammarström, Stockholm, Sweden (lennart.hammarstrom@biosci.ki.se), Piotr Heczko, Krakow, Poland (mbheczko@cyf-kr.edu.pl), Bengt Jeppson, Malmö, Sweden (bengt.jeppsson@kir.mas.lu.se), Yasuhiro Koga, Kanagawa, Japan (yasuhiro@is.icc.u-tokai.ac.jp), Lars Lundell, Stockholm, Sweden (lars.lundell@hs.se), Jennifer Madden, Dundee, United Kingdom (j.madden@dundee.ac.uk), Arne Melander, Malmö, Sweden (arne.melander@smi.mas.lu.se), Pierre Michetti, Lausanne, Switzerland (pierre.michetti@chur.hospvd.ch), Tore Midtvedt, Stockholm, Sweden (tore.midtvedt@cmb.ki.se), Marika Mikkelsaar, Tartu, Estonia (marikam@ut.ee), Göran Molin, Lund, Sweden (goran.molin@livsteki.lth.se), Elisabeth Norin, Stockholm, Sweden (elisabeth.norin@mtc.ki.se), Gregor Reid, London, Canada (greid@Iri.sjhc.london.on.ca), Theodor Rokkas, Athens, Greece (sakkor@compulink.gr), Seppo Salminen, Turkku, Finland (seppo.salminen@utu.fi), Takashi Sasaki, Kanagawa, Japan (takashi_sasaki@meiji-milk.com), Torkel Wadström, Lund, Sweden (torkel.wadstrom@mmb.lu.se), James Versalovic, Houston, U.S.A. (jamesv@bcm.tmc.edu), Agnes Wold, Göteborg, Sweden (agnes.wold@immuno.gu.se) 